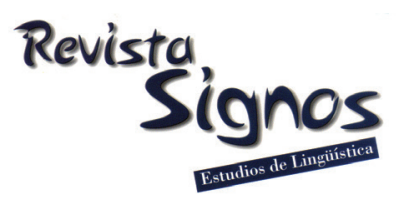

\title{
Conocimientos metalingüísticos y uso correcto de la tilde
}

\author{
Metalinguistic knowledge and correct use of accent mark \\ Luis Mamani \\ Universidad Nacional Mayor de San Marcos \\ Universidad de Ciencias y Humanidades \\ Perú \\ albertoabril115@yahoo.es
}

Recibido: 10-V-2012 / Aceptado: 22-I-2013

\section{Resumen}

El éxito ortográfico supone el manejo adecuado de determinada información. Los malos ortógrafos carecerían de esta o tendrían deficiencias al emplearla. Superar esta condición sería sencillo: los expertos deben indicarle al inexperto qué información usar y cómo emplearla. Sin embargo, hay un inconveniente: los primeros no saben exactamente qué información manejan ni cómo la utilizan. Entonces, lo crucial es identificar estos conocimientos y la manera en que son empleados. Ese es el objetivo de la presente contribución. En este empeño se ha recurrido a las técnicas de explicitación de información del análisis cognitivo de tareas. Los resultados muestran que para usar correctamente la tilde se requiere de la gestión adecuada de un conjunto de conocimientos metalingüísticos entre los que se puede mencionar la diferenciación de las clases de palabras, la determinación del número de sus sílabas, la identificación de un tipo específico de hiato, entre otros. Estos hallazgos tendrían consecuencias para la enseñanza del acento escrito.

Palabras Clave: Tareas cognitivas, ortografía, tildación. 


\begin{abstract}
Spelling success supposes the proper use of specific information. Non-experts orthographic correctors lack this information or they do have deficiencies, when using it. Overcoming this condition seems simple: the experts must pass on this knowledge by telling the novice what information to use and how. However, there is a greater challenge: experts do not know exactly what information they handle and how they use it. So it is crucial to identify these skills and how they are used. That is the purpose of this contribution. In this endeavor we have used techniques like the elicitation of information, from the cognitive task analysis. Results show that a properly use of the orthographic accent requires a proper management of a set of metalinguistic knowledge among which we can mention the differentiation of kinds of words, the determination of the number of syllables, the identification of a specific type of hiatus, among others. These findings might have implications when teaching written orthographic accent.
\end{abstract}

Key Words: Cognitive tasks, spelling, written accentuation.

\title{
INTRODUCCIÓN
}

Se afirma que la cantidad de errores en el empleo de la tilde supera la de otras áreas ortográficas (Ávila, 2002; Rico \& Vernon, 2011; Vernon \& Pruneda, 2011; Banegas \& Martínez, s. f.). Se ha señalado incluso que solo el $5 \%$ de los jóvenes españoles de dieciséis años comprende la lógica del acento escrito (Barberá, Collado, Morató, Pellicer \& Rizo, 2002). Se puede atribuir las faltas a tres tipos de causas: a) de aprendizaje: los encargados de la formación desconocen la forma en que se produce el aprendizaje de esta habilidad ortográfica; b) de enseñanza: quienes instruyen no emplean las estrategias adecuadas que propicien el aprendizaje de este dominio; c) de contenidos: los que adiestran no saben cuáles son los contenidos indispensables que permitirían al aprendiz afrontar esta tarea con éxito.

El ámbito que exploramos en el presente documento es el último de los mencionados y dentro de él nos limitamos a contenidos relacionados con el campo lingüístico, los cuales necesariamente son metalingüísticos (Díaz, 2006). Detallamos a continuación las razones de nuestra elección: a) no hemos podido hallar trabajos que indaguen por estos temas; b) entendemos que los contenidos preceden a cómo se aprende y a cómo se enseña; c) hay ciertos indicios de que los aprendices carecen de la suficiente información para la ejecución de la tarea de tildar; d) existe una estrecha relación entre las reglas de uso de la tilde y algunos contenidos pertenecientes a la gramática de la lengua; este último aspecto pertenece a nuestro campo profesional (lingüística).

Tras precisar nuestra área de interés, debemos contestar la siguiente pregunta: ¿cómo se puede determinar los conocimientos mínimos necesarios para poder aplicar correctamente la tilde? La respuesta exige considerar por lo menos dos cuestiones cruciales: superar la simple intuición y evitar el subjetivismo. Podría conseguirse 
este doble propósito con la consulta a personas que no se equivocan al marcar la tilde por ser las más idóneas para proporcionar estos valiosos datos. Sin embargo, los expertos presentan una peculiaridad: por paradójico que parezca, hay algunas evidencias que nos indican que ellos no conocen a cabalidad toda la información que manejan para poder realizar esta labor ortográfica. Revisemos a continuación algunos de estos indicios: los especialistas sustentan la enseñanza de esta habilidad solo en dar a conocer las reglas de la Real Academia Española (RAE) con lo cual asumen que eso es lo único que se necesita para aplicar correctamente la tilde; por esta razón, les causa mucha extrañeza la cantidad de errores en que incurren sus alumnos, pues entienden que en esta parte de la ortografía castellana las reglas son más sistemáticas y sencillas (Pérez, 1994; Zayas, 1995; Portilla, 2003; Serna 2004; Pujol, 2005).

El desconcierto de algunos de ellos es tan grande que llegan a afirmar que la causa principal de estas equivocaciones es el desinterés de los estudiantes hacia este asunto (Pérez, 1994; Ávila, 2002). Entonces, en esencia, se piensa que para ser experto en el uso de la tilde solo se requiere conocer exhaustivamente las reglas de la RAE. Sin embargo, esto no se condice con la realidad, porque tras culminar su instrucción básica obligatoria, más o menos homogénea, los aprendices muestran una tremenda heterogeneidad en el manejo de la tilde con un predominio de los que se equivocan bastante. Si todos recibieron idéntico contenido normativo, algo distinto a dicho dominio debe estar decidiendo los logros de pocos y los yerros de la mayoría.

El valor de los conocimientos antes mencionados y el empleo poco explícito que de ellos hacen los expertos determinan los dos objetivos de la presente investigación: a) explicitar dicha información; b) mostrar la manera en que es empleada al momento de escribir el acento ortográfico. A su vez, conocer todo ello nos permitirá lo siguiente: a) acercarnos a la comprensión de los procesos mentales subyacentes al trazado de esta marca ortográfica; b) entender por qué esta tarea resulta tan difícil para muchas personas; c) sugerir la enseñanza de estos imprescindibles contenidos; d) proponer, incluso, una secuenciación de contenidos para conseguir una competencia paulatina en el empleo de las reglas del acento gráfico.

La exploración del empleo de los conocimientos subyacentes a la ejecución de alguna actividad ha sido emprendida por psicólogos dedicados al análisis de tareas. En vista de que en los últimos años se ha conseguido resultados significativos mediante los procedimientos del análisis cognitivo de tareas (ACT), hemos recurrido a ellos en el trabajo que estamos exponiendo; este lo hemos organizado del siguiente modo: en primer lugar, presentamos las ideas que fundamentan el ACT; en segundo lugar, situamos la tilde dentro de la ortografía de la lengua española; enseguida, exponemos la manera en que hemos aplicado el ACT; después, valiéndonos de la aplicación del ACT, presentamos tanto los conocimientos que se requieren para usar correctamente el acento escrito como la forma en que estos estarían siendo gestionados por la mente al momento de aplicar la tilde; finalmente, damos a conocer las conclusiones a las que hemos arribado. 


\section{Marco de referencia}

\subsection{Antecedentes}

Desconocemos la existencia de investigaciones cuyo objetivo haya sido determinar los conocimientos implicados en el dominio del acento escrito. Portilla (2003) y Zayas (1995) mencionan en sus trabajos algunos contenidos metalingüísticos que se requieren al escribir la tilde; sin embargo, ello no es exhaustivo debido a que ambos autores se centran en aspectos de enseñanza. El análisis de los errores acentuales en textos producidos por aspirantes a la universidad que realiza Pujol (2005) concluye, sin los argumentos necesarios, que son causados por su insuficiente competencia metalingüística. Borzi y García (2006) adoptan una perspectiva cognitiva en su estudio sobre el manejo del acento escrito; pero su interés no es la información necesaria para su empleo correcto, sino algunas razones pragmáticas que inducen su uso incorrecto.

\subsection{El análisis cognitivo de tareas}

Averiguar del experto qué información metalingüística maneja y cómo la utiliza al momento de graficar la tilde es una tarea que no está al alcance de la lingüística. Por tal motivo, se tuvo que echar mano de recursos que la psicología ha usado satisfactoriamente en situaciones similares. Nos referimos específicamente al análisis de tareas. En general, el supuesto de cualquier propuesta enmarcada en el análisis de tareas es que el diestro ejecuta con éxito las labores en las que es considerado como tal sin tener claro cómo es que las realiza (Chipman, Schraagen \& Shalin, 2000). Y precisamente, el objetivo del análisis de tareas es hacer explícitos esos conocimientos que escapan a la consciencia del experto.

Dentro de las varias propuestas que existen al amparo de esta perspectiva, nos hemos decidido por el ACT que proponen Crandall, Klein y Hoffman (2006). La principal razón de nuestra elección es que apreciamos mayor objetividad en los procedimientos de explicitación de los conocimientos subyacentes a la ejecución de alguna tarea. El ACT escogido constituye un conjunto de métodos que se usan para estudiar y describir el conocimiento y el razonamiento. Tiene el propósito de capturar el modo en que la mente trabaja durante la ejecución de alguna tarea. Ello significa aprehender lo que los diestros conocen y creen sobre un determinado dominio: conceptos, principios, eventos. Es decir, todo aquello que los convierte en entendidos en la materia.

La aplicación del ACT exige el cumplimiento de una serie de actividades previas a la recolección de los datos. Ello ha sido denominado por los autores como acciones de preparación y encuadre. Este conjunto de labores permite orientar de mejor manera el acopio de los datos y su posterior análisis. El objetivo que se persigue con el enmarcado es que los analistas tengan suma claridad sobre ciertos aspectos que justifican la realización del ACT. Deben saber con precisión qué necesidades se desea 
satisfacer (diseño de estrategias de entrenamiento, comprensión de errores, reducción de riesgos), qué documento se debe entregar tras finalizar el trabajo, con qué gente se puede trabajar, qué tipo de cognición se necesita conocer, cuál es el punto de partida (si se parte desde cero o hay material que se puede aprovechar).

Para desentrañar la información que la mente procesa durante la realización de alguna actividad, se recurre a diversos medios: entrevistas a expertos en el área de estudio, observaciones a estos mientras efectúan sus tareas, manuales de instrucciones, informes de los peritos, autorreportes de los especialistas sobre cómo ejecutan sus acciones, análisis de incidentes, análisis de errores.

Por otra parte, la propuesta por la que aquí hemos optado comprende la minuciosa elaboración del mapa conceptual correspondiente a la tarea que es objeto de estudio. Este recurso gráfico se diseña con toda la información conseguida en el proceso de explicitación del conocimiento. Si tenemos en cuenta el propósito que se persigue con el ACT, la contribución del mapa conceptual es doble: a) permite poner de manifiesto el conocimiento de los peritos; b) hace posible representarlo de manera gráfica. Además, no se debe perder de vista que la elaboración del mapa conceptual le permite al analista alcanzar claridad sobre lo que desea expresar. Y esto es así, porque graficar este instrumento, en la práctica, ya supone el análisis de datos.

Luego de haber recopilado la información pertinente, se procede a su interpretación. En esta fase, el supuesto es que los conocimientos que poseen los diestros para la ejecución de sus tareas se revelan en algunos hechos observables y sistemáticos. Entonces, la forma en que los expertos llevan a cabo sus actividades, las secuencias que siguen y las instrucciones que dan a los novatos son algunos de los aspectos que deben ser interpretados. En el caso de los no diestros, lo que debe examinarse son sus errores sistemáticos, las circunstancias en las que más aciertan y las situaciones en las que más yerran.

\subsection{El lugar de la tilde en la escritura del castellano}

Lacon y Ortega (2008), citando a Mata (1997), refieren que el uso del código gráfico compromete procesos y actividades cognitivos que a su vez implican varios subprocesos jerárquicamente organizados en cuyo máximo nivel está el control del proceso mismo. La actividad ortográfica supone factores cognitivos y lingüísticos (Pujol, 1999). El factor lingüístico se halla involucrado, porque las unidades del código alfabético guardan estrecha relación con elementos fónicos de la lengua. Existe actividad cognitiva, pues el sujeto debe gestionar una serie de recursos mentales para recuperar la forma ortográfica correcta de las palabras y usar el código alfabético.

Lingüísticamente, entonces, la tilde debe ser vista en sus dos vertientes: a) como una unidad del código escrito que guarda relación con algún constituyente de la lengua oral; b) como un elemento al que le corresponde un lugar dentro de todo el sistema 
de escritura del castellano. En lo que concierne a lo primero, el constituyente con el que se vincula la tilde es el acento primario. Este se confiere a las palabras mediante el principio de asignación acentual el cual solo puede operar luego de que las palabras ya han sido formadas (Guitart, 2004). Considerada en su pertenencia al código gráfico, la tilde forma parte de la ortografía de la palabra (Martínez de Sousa, 2003). Las letras también están comprometidas en la representación gráfica de las palabras, pero estarían en un nivel distinto que el de la tilde: en el proceso de codificación, las operaciones cognitivas que subyacen a la marcación de la tilde ocurrirían con posterioridad a la consecución de la representación grafemática de la palabra. Esto guarda estrecha relación con la forma como, en el ámbito fónico, las palabras reciben su acento primario: este solo puede ser asignado después de haber sido conformada la palabra en sus fonemas segmentales, no antes ni durante. El hecho de que algunos alumnos hayan escrito <asín> y otros < asin> (vulgarismo de 'así') (Pujol, 1999) es una evidencia que apoya nuestra afirmación, pues todos los que escribieron de la primera o de la segunda forma sabían que se trataba de la única palabra /asin/ (forma fonólogica) cuyo significado es "De esta o de esa manera..." Otra evidencia podría ser aquella en la que la misma persona en el mismo renglón haya redactado "empezo a pelear con los canivales y mato al rey de los caníbales" (Duque, s. f.); en este caso, la actividad escrituraria se habría centrado casi exclusivamente en relacionar las letras con los fonemas segmentales, y la omisión de las tildes no se habría producido por alguna carencia informativa, sino simplemente porque el proceso cognitivo correspondiente a la marcación de la tilde no habría llegado a su culminación por acontecer después de la consecución de la representación de las palabras en sus correspondientes grafemas. La tilde no tiene representación independiente de las letras, pero las letras no requieren de la tilde para poder conseguir su representación escrita.

Desde el punto de vista cognitivo, marcar el acento escrito en una palabra o no hacerlo presupone manejar información proveniente de la gramática de la lengua (v. g. acento, clases de palabras) y del código alfabético (v. g. sílaba ortográfica, grafema). Si tenemos en cuenta los modelos de procesamiento ortográfico que reseña Pujol (1999), suponemos que la decisión de escribir la tilde en alguna palabra es una operación posterior a su recuperación grafemática por lo que acontecería en el almacén de la memoria de trabajo.

\section{Aspectos metodológicos}

El trabajo que hemos realizado con el ACT ha supuesto tres etapas bien definidas: a) la planificación o, en términos del ACT, el enmarcado; b) el acopio de datos; c) la interpretación de lo recopilado.

Gracias a que se ha cumplido con la primera etapa, el ATC ha recibido una orientación peculiar que se apreciará en la segunda fase. Para la recolección de la información, hemos recurrido a diversas fuentes. La que ha tenido mayor importancia 
ha sido la conformada por documentos que analizan los yerros de los aprendices en la aplicación de la tilde. En segunda instancia, hemos considerado el material bibliográfico relacionado con la enseñanza y el aprendizaje del acento ortográfico. También hemos tenido en cuenta los errores cometidos por los alumnos. En cuanto a los especialistas, se determinó que quienes podían ser observados en su labor y ser consultados sobre ella serían lingüistas dedicados a la enseñanza de la ortografía de la lengua castellana debido a que ellos poseen los conocimientos suficientes acerca de la gramática de esta lengua y pueden intervenir con propiedad sobre las nociones lingüísticas involucradas en el manejo de la tilde. Se decidió analizar su desempeño docente en los primeros ciclos de estudios universitarios, puesto que el alumnado que ha llegado a este nivel ya ha abordado de manera suficiente todo lo concerniente al acento escrito. Se observó la labor instructiva de tres profesores (P. Aguilar, M. Rosales \& R. Quintanilla) en el segundo semestre de 2011 y se tuvo entrevistas con ellos en enero de 2012.

Finalmente, a partir de estos datos, se ha emprendido la tarea de la identificación de los conocimientos que entran en juego al aplicar la tilde. En esta fase, de acuerdo con el ACT, la primera acción que se realizó fue la elaboración del mapa conceptual correspondiente, en el que mostramos los conceptos imprescindibles relacionados con la tilde y las relaciones existentes entre estos.

\section{Aplicando el ACT}

\subsection{Cuestiones de enmarque}

La realización del ACT ha tenido un doble propósito: a) determinar los conocimientos metalingüísticos que exige el empleo correcto del acento escrito; b) establecer cómo estos son gestionados al aplicar la tilde. No se ha partido de cero, pues se ha utilizado convenientemente el material ya publicado. Los resultados son expuestos en el presente documento académico. Conocer esta información permitirá proponer cambios en la enseñanza de esta área de la escritura con el propósito de mejorar la competencia ortográfica de los aprendices.

\subsection{Los conocimientos necesarios}

En primera instancia, para guiar nuestro trabajo, presentamos en la Figura 1 el mapa conceptual relacionado con el manejo de la tilde.

\subsubsection{El silabeo}

Los datos obtenidos con el ACT nos permiten afirmar que la colocación de la tilde en cualquier palabra implica el conocimiento explícito del silabeo fónico y la identificación de la sílaba tónica. Si alguna persona no puede realizar estas actividades previas no podrá aplicar la tilde correctamente. A este convencimiento ya habían 
llegado los especialistas entrevistados y ello también se encuentra remarcado por Portilla (2003), Pujol (1999) y Zayas (1995). Asimismo, Rico y Vernon (2011) dan cuenta de investigaciones en las que se señala la trascendencia de este saber para el empleo correcto de la tilde por parte de los niños. La capacidad de efectuar el silabeo fónico y el reconocimiento de la sílaba tónica constituirán nuestro conocimiento metalingüístico A.

\section{¿Qué conocimientos \\ se requieren para aplicar la tilde?}

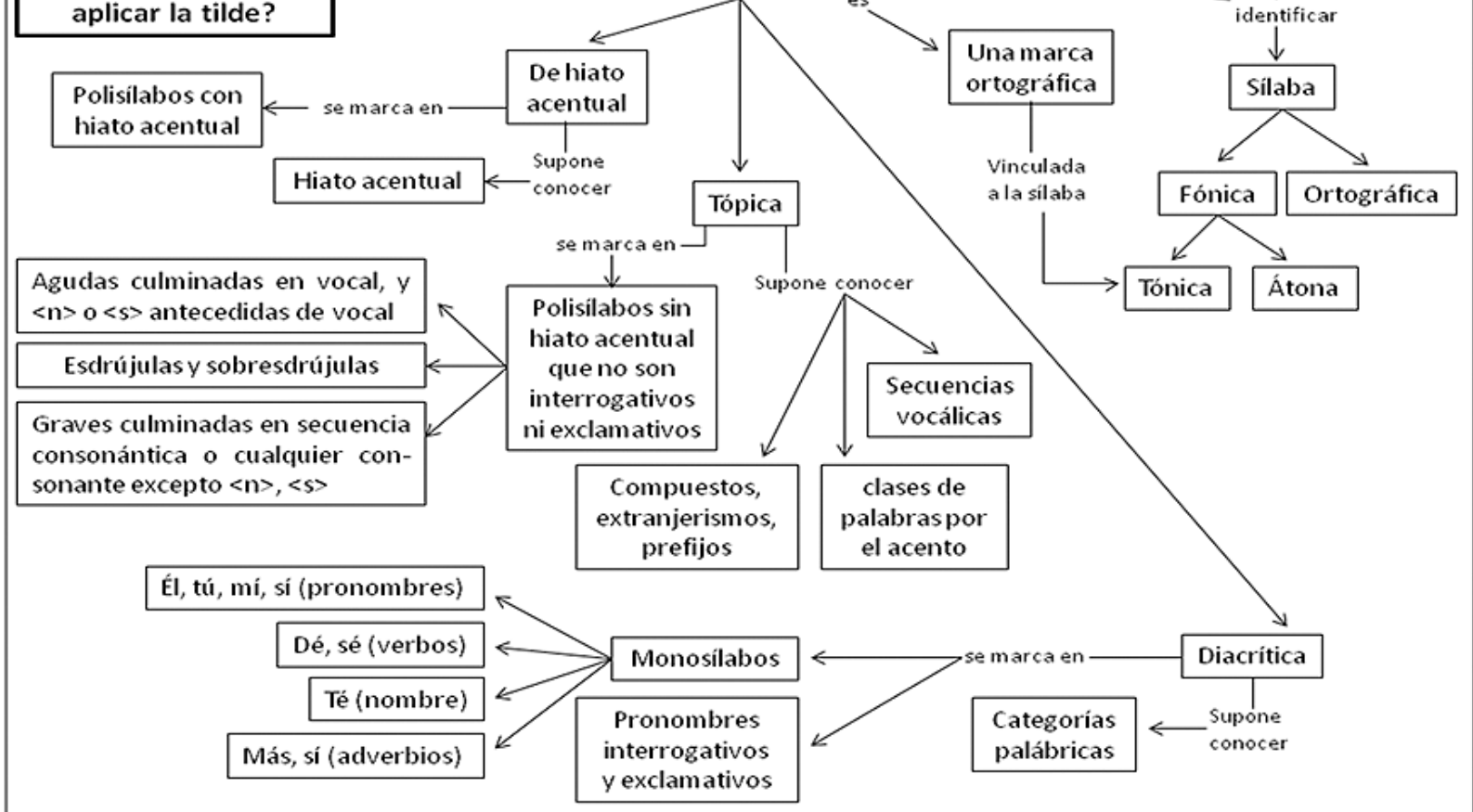

Figura 1. Mapa conceptual de la tilde.

Aguilar, Rosales y Quintanilla, nuestros entrevistados, al impartir sus clases tienen especial preocupación por explicar la diferencia entre el acento y su representación gráfica, o lo que es lo mismo, entre el acento y la tilde. Sus motivos no admiten discusiones: no a todas las sílabas acentuadas les corresponde tilde. Portilla (2003) señala que la primera tarea del docente que aborde el tema del acento escrito será establecer esta separación de nociones. En nuestro mapa conceptual, como se puede apreciar, no hemos hecho otra cosa que representar tal distinción. Este será el conocimiento metalingǘrstico $\mathrm{B}$.

A los conocimientos A y B, hay que agregar la capacidad que debe tener el hablante sobre el silabeo ortográfico. Conocer esto último es indispensable, 
debido a que hay palabras en las que los límites de sus sílabas fónicas no coinciden exactamente con los de sus correspondientes sílabas ortográficas: el silabeo fónico de la palabra taxi es [tak-si] y su silabeo en la escritura es $<$ ta-xi $>$; fónicamente, la palabra cohesión se silabea [ko-e-sjon] y si la escribimos podría silabearse $<$ cohe-sión> o <co-he-sión>, pero por las reglas de la RAE solo la última es correcta. En casos como estos se prioriza la sílaba ortográfica, es decir, es esta noción la que cuenta en última instancia para trazar este tipo de virgulilla. Además, no debemos olvidar "que algunas combinaciones vocálicas se consideran siempre diptongo a efectos de acentuación gráfica, sea cual sea su pronunciación" (RAE, 2005: 636). La RAE seguramente se refiere a palabras como $<$ huir $>$ o $<$ huías $>$ que desde el punto de vista de la escritura deben ser silabeadas como <huir $>$ (monosílabo) y $<$ huí-as $>$ (bisílabo) independientemente de su pronunciación silábica: sea como bisílabo ([u. ír]) y trisílabo ([u.í.as]) (Guitart, 2004) o como monosílabo ([uír]) y bisílabo ([uí.as]). Asimismo, debe tenerse presente que el silabeo ortográfico es el único pertinente para tildar o no las palabras compuestas (<cor-ta-ú-ñas $>,<$ con-tra-hí-lo $>,<$ so-brehi-lar) y las palabras con prefijos ( $<$ re-hí-la $>,<$ rein-ser-tar $>$, $<$ pre-ín-ca $>$ ). Sintetizando esta parte: para usar correctamente la tilde, es de vital importancia poder efectuar el silabeo ortográfico. El nombre que le vamos a asignar a este requisito será el de conocimiento metalingüístico C. Para culminar este apartado, debemos señalar que es casi seguro que aquellos que escribieron <enamorarón $>,<$ sentarón $>,<$ caminarón $>$ (Duque, s. f.), <debia $>$, <prácticar $>,<$ despues $>$, <hablarón $>$ (Portilla, 2003), <imagén $>$, $<$ imagénes>, <cambién>, < régiones> (Pujol, 2005) tienen deficiencias en estos dos saberes fundamentales; es evidente que ellos no pudieron relacionar la sílaba tónica con la tilde. Suponemos que en similar situación están quienes escribieron $<$ huí $>$, $<$ fuí> que habrían identificado erróneamente estas palabras como bisílabas agudas terminadas en vocal por lo que procedieron a colocar la tilde.

\subsubsection{Polisílabos y monosílabos}

La lectura de nuestro mapa conceptual nos señala que, para aplicar bien la tilde, nuestra mente debe efectuar una bipartición de las palabras según el número de sus sílabas: a) monosílabos; b) polisílabos. La razón de esta división es sencilla: unas y otras palabras son sometidas a procesos de tildación bien diferenciados. Es obvio que para graficar la tilde en el pronombre personal <mí> (es para mí) y no hacerlo en el determinante posesivo $<\mathrm{mi}>$ ( $\mathbf{m i}$ amigo salió) se sigue un criterio diferente del seguido en palabras como $<$ cráter $>$ o $<$ bisturí $>$. La relevancia de este conocimiento sería la razón por la que Aguilar y Rosales inicien el tema específico del acento ortográfico con la identificación de estas dos clases de palabras; también sería la causa para que la RAE (2010) tenga un apartado específico para esta señal gráfica en las palabras de varias sílabas. Entonces, sobre la base de los conocimientos A, B y C, se debe poder distinguir entre un polisílabo y un monosílabo. Este será el conocimiento metalingǘstico D. Es probable que quienes hayan escrito equivocadamente $<$ fué $>$, $<$ dió> , <vió>, <bién> (Pujol, 2005) no hayan logrado establecer esta diferenciación. Antes de finalizar este apartado, es preciso recordar que hay un reducido grupo de 
polisílabos que se tilda siguiendo el criterio aplicado para los monosílabos, lo cual será analizado con mayor detalle cuando se aborde el uso de la tilde en las palabras de una sola sílaba.

\subsubsection{Polisílabos}

Para aplicar correctamente la tilde en los polisílabos, no basta con que los hayamos podido reconocer como tales y haberlos diferenciado de los monosílabos; esta insuficiencia se debe a que no hay un criterio único para tildarlos. Por tal motivo, este grupo de palabras debe ser subdividido en dos: a) las que presentan un tipo específico de hiato; b) las que carecen de él. El hiato al que nos referimos es el que se representa ortográficamente por una $<\mathrm{i}>$ o una $<\mathrm{u}>$ pronunciadas con intensidad o acento, que se presentan antes o después de $\left.\left.\left.<_{\mathrm{a}}\right\rangle,<_{\mathrm{e}}\right\rangle,<_{\mathrm{O}}\right\rangle$ pronunciadas sin fuerza de voz (v. g. actúo, caída, sonreír). Portilla (2003) emplea el nombre de 'hiato acentual' para esta secuencia de vocales que pertenecen a sílabas diferentes y el de 'hiato simple' para cualquier otra combinación vocálica heterosilábica distinta de esta que acabamos de describir (v. g. poeta, cooperar, héroe, chiita, duunviro). Estas denominaciones también se usan en un manual de enseñanza (Villafana, Chacón, Conde \& Quintanilla, 2009). La asignación de estos nombres nos parece acertada y la adoptaremos en el presente trabajo. Entonces, retomando el tema, la distinción que se tendrá que realizar dentro del conjunto de las palabras polisílabas será entre las que contienen hiato acentual y las que estén privadas de él. La razón de esta separación es que el criterio que se usa para tildar los polisílabos con hiato acentual no es el mismo que se sigue para acentuar ortográficamente los polisílabos que no tienen esta secuencia vocálica. La capacidad de establecer esta diferencia será el conocimiento metalingüístico E.

Tener deficiencias al establecer esta diferenciación estaría ocasionando que la cantidad de errores en los hiatos acentuales supere largamente la correspondiente a otros grupos de palabras (Portilla, 2003; Pujol, 2005). Lo más probable es que los aprendices que hayan omitido la tilde en $\langle$ dia $\rangle,\langle$ situa $\rangle$, $<$ energia $\rangle,\langle$ actua $\rangle$, $<$ habia $>$, <oido $>$ (Pujol, 2005) hayan pensado que estaban ante palabras graves terminadas en vocal, razón por la que decidieron no tildarlas. Uno de nuestros entrevistados, Quintanilla, afirma específicamente que la omisión de la tilde en estas palabras obedece a que su escritura contradice las reglas generales. Por su parte, Aguilar y Rosales manifiestan que en sus clases enfatizan la identificación de este tipo de hiato.

Después de haber subdividido los polisílabos en estos dos grupos, se puede hablar de la aplicación de la tilde en estas palabras. Como se sabe, para marcar la tilde en los polisílabos con hiato acentual se debe tener presente una única regla: se tílda siempre la vocal cerrada $(<\mathrm{i}>,<\mathrm{u}>)$ pronunciada con acento si aparece antes o después de una vocal no cerrada $\left.\left(<_{\mathrm{a}}\right\rangle,<\mathrm{e}>,<_{\mathrm{O}}>\right)$ pronunciada sin fuerza de voz. En otras palabras, se tilda la vocal cerrada de todo hiato acentual sin excepción alguna. Cualquier otra 
secuencia vocálica distinta a esta combinación específica ocasiona que la palabra correspondiente salga automáticamente del ámbito de esta regla y pase a ser sometida a otras normas pues, o bien se trata de un polisílabo sin hiato acentual, o bien se trata de un monosílabo. Es válido afirmar también que este grupo específico de palabras se acomoda perfectamente a un tipo particular de tilde, que, como se puede observar en nuestro mapa conceptual, hemos etiquetado como 'tilde de hiato acentual', denominación que es puramente descriptiva. Hay que indicar que en el sur del Perú tiene arraigo la denominación 'tilde robúrica' (véase, por ejemplo, Cano 1975). En Bolivia, Sosa (2007) recurre al nombre 'tilde isócrona'. La RAE, en este caso, no habla de un tipo especial de tilde, pero tiene un apartado titulado "La acentuación gráfica de las palabras con secuencias vocálicas" (Sosa, 2010), lo cual nos parece inadecuado, porque no existe una clase de acento escrito aplicable a todas las palabras con secuencias vocálicas. Las palabras que contienen diptongo(s), las que presentan triptongo y las que contienen hiato(s) simple(s) se someten a las, denominadas así por la RAE, reglas generales de acento gráfico; pero las palabras con hiato acentual no están comprendidas en estas reglas. Si esto es así, no tiene mayor sentido haberles dedicado un lugar aparte a todas ellas. Lo que se tendría que haber hecho es separar los polisílabos con hiato acentual y agrupar las que contienen los otros tipos de secuencias vocálicas con el resto de polisílabos. Nuestra objeción adquiere relevancia cuando leemos: "Solo constituyen una excepción a las pautas generales las palabras que contienen un tipo particular de hiatos, como se detallará más adelante" (RAE, 2010: 233). En esa excepción están comprendidas las palabras que contienen hiato acentual las cuales se tildan solo por el hecho de contener este tipo de hiato. Si estos polisílabos se tildan independientemente de cualquier otra consideración, es legítimo hablar de una clase específica de tilde. Saber que se tilda siempre la vocal cerrada de los hiatos acentuales será nuestro conocimiento metalingüístico F.

Es pertinente hacer notar que la tilde que se marca en las palabras que contienen hiato acentual es la que menos conocimientos metalingüísticos exige: desde el A hasta el F. La complejidad continúa siendo la menor incluso si se pretende tratar en esta parte el tema de las restantes secuencias vocálicas intrapalábricas: diptongo, triptongo y hiato simple. Hasta aquí lo concerniente a las palabras con hiato acentual.

En lo que respecta a los polisílabos sin hiato acentual, hay que indicar que se someten a las reglas de una tilde específica que, como se puede apreciar en nuestro mapa conceptual, hemos rotulado como 'tópica'. Al recurrir a este nombre solo estamos continuando con una práctica que acontece en algunos lugares del Perú y Bolivia (Academia Boliviana de la Lengua, 2011). No estamos de acuerdo con la denominación, ampliamente conocida, de 'tilde general' pues no se trata de reglas generales de aplicación del acento ortográfico en cualquier palabra. De lo que se trata en realidad es solo de un conjunto de reglas aplicables a un grupo definido de palabras. La RAE incurre en un error al afirmar que las "reglas de acentuación gráfica 
de las palabras polisílabas se aplican en función de si son agudas, llanas, esdrújulas o sobresdrújulas" (RAE, 2010:231), puesto que hay polisílabos que se tildan atendiendo a otras consideraciones: en primer lugar, están las palabras con hiato acentual; y en segundo lugar, los polisílabos que son partículas interrogativas o exclamativas. Por ello la afirmación de la RAE debió especificar que se trata solo de polisílabos sin hiato acentual y que no son partículas interrogativas o exclamativas.

Para poder aplicar la tilde en estas palabras, que carecen de hiato acentual y que no son interrogativas ni exclamativas, es conditio sine qua non saber cómo clasificarlas según el acento. La capacidad de establecer esta clasificación será para nosotros el conocimiento metalingüístico G. Para los hablantes, se trata solo de un reconocimiento, puesto que, como ya se señaló, después de que la palabra tomó forma a través de sus fonemas segmentales, la gramática le asigna su acento primario, razón por la cual aquellos ya saben pronunciar las palabras correspondientes. Lo que deben hacer ahora los hablantes es rotular como agudas las palabras acentuadas en la última sílaba; como graves las acentuadas en la penúltima sílaba, y así consecutivamente. Además, y esto se puede apreciar en nuestro mapa conceptual, para poder usar la tilde en estas palabras, se requiere un conjunto de conocimientos metalingüísticos como para poder lidiar con compuestos (puntapié, portaguión, ítalo-peruano, decimosexto); prefijos (semidiós, rehízo); extranjerismos crudos (standard, blue jean, scanner) y castellanizados (estándar, bluyín, escáner); diptongos (lingüista, lingüística, ruido, cuídate), triptongos (hioides, averigüéis) y hiatos simples (odriista, priismo, friísimo). También debe considerarse el tema de los adverbios terminados en -mente en los que puede percibirse dos acentos primarios. Y todo ello porque son esas nociones las que están implicadas en la aplicación de la tilde en estas palabras. Por comodidad expositiva, proponemos agrupar todo este saber bajo el nombre de conocimientos $\mathrm{H}$. A esto, evidentemente, habrá que agregar el dominio de las reglas de tildación de las palabras agudas, graves, esdrújulas y sobresdrújulas. Esto último será considerado por nosotros como conocimientos I.

\subsubsection{Monosílabos}

Si tras el silabeo fónico y ortográfico una palabra es reconocida como monosílaba, deberá ser sometida a las reglas de la tilde diacrítica. Y de acuerdo con la normativa vigente, esta tilde se aplica solo a ocho monosílabos y a las partículas interrogativas y exclamativas (RAE, 2010). Como ya se señaló, algunas de estas palabras interrogativas y exclamativas son polisílabas, pero para efectos de la tilde no cuenta el número de sus sílabas, sino solo su pertenencia a cualquiera de las dos clases de palabras mencionadas. La tilde diacrítica nos permite, por ejemplo, diferenciar 'de' (preposición) de 'dé' (verbo). Precisamente, Vernon y Pruneda (2011) atribuyen a Julio Casares (1952) haber identificado determinadas funciones que le corresponden a la tilde. Así, gracias a las funciones prosódica, diacrítica y tonal de la tilde, se puede distinguir en el sistema (lengua) entre pares de palabras: mí (pronombre posesivo) y 
mi (determinante), sí (pronombre reflexivo o adverbio de afirmación) y si (conjunción condicional o conjunción completiva), cuál (pronombre interrogativo o pronombre exclamativo) y cual (pronombre relativo), etc.; de este modo, según la perspectiva de Casares, si no hubiera tilde en uno de los pares de estas palabras, los que actuamos como decodificadores no podríamos identificar las categorías a las que pertenecen y tendríamos problemas para la interpretación de los enunciados correspondientes. Por su parte, Pujol (2005) afirma que el acento gráfico posee dos funciones: a) señalar la vocal que tiene acento de intensidad; b) distinguir la categoría gramatical y semántica de determinadas palabras. La RAE (2010) usa las denominaciones de prosódica y diacrítica para estas mismas funciones. Es evidente que al señalar estas funciones de la tilde se ha adoptado el punto de vista del decodificador, pues es este el que al apreciar la tilde en estas palabras podrá identificar sin problemas las clases a las que corresponden. Pero ¿qué ocurre si analizamos estas funciones no desde la perspectiva del decodificador, ni considerando el código mismo, como entendemos que lo hacen Casares, Pujol y la RAE, sino desde el punto de vista del que va a codificar, del que va a escribir? Si cambiamos de punto de vista, lo que sucede es que hallamos exactamente lo complementario a lo señalado por estos autores: que para poder aplicar correctamente esta clase de tilde es imprescindible que en la mente del codificador haya tenido que establecerse con anterioridad esta diferenciación entre las clases de palabras en cuestión; o sea, el codificador podrá decidir si escribe o no la tilde en alguna de estas palabras solo si ha reconocido previamente la categoría a la que aquellas pertenecen. Es altamente probable que los alumnos que debieron haber redactado $<$ más $>,<$ sí $>$, <él $>$, $<$ sé $>,<$ dé $>,<$ mí $>$, $<$ tú $>$ hayan optado finalmente por escribir estos monosílabos sin tilde (Pujol, 2005) a causa de una deficiente identificación de las clases de palabras.

Entonces, la pericia en esta clase de tilde supone que el hablante reconozca varias clases de palabras: pronombre interrogativo, pronombre exclamativo, determinante, preposición, adverbio, verbo. La diferenciación entre una y otra de estas categorías será nuestro conocimiento metalingüístico $\mathrm{J}$.

\subsubsection{Competencia grafémica}

Al revisar los errores de los aprendices, hay algunos que no logran explicarse en el marco de nuestro mapa conceptual. Nos referimos a palabras registradas como $<$ matís $>$ o <perdís> que hemos podido apreciar esporádicamente en textos producidos por algunos de nuestros alumnos. Este tipo de equivocación, que solo podría presentarse en dialectos seseantes como el peruano, no puede ser achacado al desconocimiento de las reglas de la tilde tópica, sino que debe atribuirse al desconocimiento de la representación grafémica de esas palabras por parte del novato, pues es evidente que, según su razonamiento, se trataría de palabras agudas terminadas en $<\mathrm{s}>$. Lo que pone en evidencia este tipo de yerro es que el uso correcto del acento escrito entraña el dominio cabal de la representación grafémica de las palabras. Esto constituirá nuestro conocimiento metalingüístico $\mathrm{K}$. 


\subsection{La regulación metacognitiva}

Hay alumnos que demuestran un saber pormenorizado de las reglas vigentes del acento ortográfico y también de los conocimientos metalingüísticos ya señalados a lo largo de este trabajo; sin embargo, continúan cometiendo errores. Algunos investigadores afirman que este fenómeno se debe a la falta de reforzamiento en el conocimiento de la reglas de este rasgo gráfico (véase, por ejemplo, Portilla, 2003). Pero ¿cuál podría ser la respuesta considerando el ACT? Veamos: tanto experimentados como inexpertos poseen idéntica información, pero mientras que los primeros no se equivocan al graficar la tilde, los segundos sí (y algunos, muchísimo). Entonces, ¿qué es lo que hace la diferencia? La respuesta a esta pregunta nos exige tener en cuenta algunos hechos: a) se ha observado que cuando los aprendices enfrentan cada tipo de tilde por separado tienen un mejor desempeño que cuando deben acentuar ortográficamente palabras que pertenecen a todas las tildes; el profesor Aguilar corroboró esta aseveración en la entrevista correspondiente; b) en las pruebas que incluyen todas las clases de palabras, el ámbito donde el número de errores aumenta notablemente es en el correspondiente a la tilde en los hiatos acentuales (Portilla, 2003; Pujol, 2005); c) la profesora Rosales afirma que cuando está explicando la tilde en las palabras con hiato acentual, los alumnos parecen entender todo a la perfección, lo cual se confirma en los ejercicios del momento donde prácticamente no hay equivocaciones; pero algunas horas después estas afloran con facilidad. Esta diferencia de rendimientos no ocurre en absoluto en el caso de los expertos. Esto quiere decir que la diversidad de palabras por tildar no causa ninguna interferencia en el manejo de la información normativa y metalingüística que posee el experto, pero sí en la que tiene el aprendiz. Este contraste en el desempeño ortográfico de peritos y bisoños nos estaría indicando que no basta con poseer determinados conocimientos normativos y metalingǘsticos, sino que es necesario saber administrarlos. Y estas operaciones de administración de los conocimientos ya no son de índole lingüística ni metalingüística, sino de naturaleza metacognitiva. Se trata específicamente de operaciones de regulación metacognitiva las cuales se encargan de las labores de monitoreo y de control cognitivos (Fernández-Duque, Baird \& Posner, 2000). La regulación metacognitiva es requerida en situaciones de conflicto o de complejidad cognitivos, es decir, cuando los conocimientos con los que se cuenta para la ejecución de alguna tarea presentan ciertas incongruencias. Tratándose de la tilde, nos hallamos ante información incoherente en el caso de las partículas interrogativas y exclamativas polisílabas, las que por contener varias sílabas deberían tildarse según las reglas de la denominada tilde general; sin embargo, se tildan según las reglas de la tilde diacrítica. También habría contenidos conflictivos, o por lo menos complejos, en el caso de las palabras con hiato acentual. El alumno, entonces, tiene más o menos el siguiente problema: al encontrarse, por ejemplo, con la partícula interrogativa /donde/, tiene en cuenta que la tilde general se aplica a las palabras agudas, graves, etc. bajo ciertas condiciones (con algunas excepciones) y que la tilde diacrítica se aplica a algunos monosílabos; entonces, debido a que el calificativo de 'general' tiene el peso que su 
significado le otorga y a que el alumno ha tenido un prolongado y casi exclusivo contacto con esta clase de tilde, opta por no tildar esta palabra pues la considera como grave terminada en vocal. Ante tal partícula, la mente del experto recupera oportunamente la información relevante para procesarla y escribe $<$ dónde $>$ a pesar de ser una grave terminada en vocal, pues sabe que esta palabra pertenece al ámbito de la tilde diacrítica y no al de la tilde tópica. Esta forma de proceder se debe a que el experto maneja operaciones de regulación cognitiva.

Si este procedimiento del especialista es tan importante para la aplicación del acento escrito, debemos hallar la forma de representarlo. Los indicios para su esbozo podemos encontrarlos en la heterogeneidad de la competencia de los aprendices ante diferentes situaciones, que hemos explicado en el párrafo anterior, lo cual contrasta con la homogeneidad de rendimiento de los expertos ante cualquier situación. Se puede advertir otra pista al preguntarnos por las operaciones mentales que pretenden conseguir Portilla (2003) con su llamado al reforzamiento o Rosales con la repetición escrita de las palabras objeto de equivocación por parte de los alumnos. O sea ¿qué tienen en mente ellas para estar convencidas de que la realización de una u otra actividad es la que ayudará a los no diestros en el manejo de la tilde? Nuestra respuesta es que lo que ambas están buscando es que los novatos hagan suyo el procedimiento que ellas usan para aplicar la tilde. Lo que no hacen los inexpertos, pero sí ellas, como expertas que son, es hacer 'pasar' todas las palabras por las tres clases de tilde que hemos presentado en el presente trabajo: tópica, de hiato acentual y diacrítica. Pero esto es solo una parte de toda la gestión de la información. Por todo lo expuesto hasta el momento, estamos en capacidad de afirmar que lo que hacen los expertos antes de aplicar la tilde es silabear fónicamente las palabras, identificar la sílaba tónica (acento) y efectuar el silabeo ortográfico. Enseguida, hacen ingresar las palabras a un primer dispositivo, el de la tilde tópica, en el que solo se reconoce polisílabos sin hiato acentual y que no son pronombres interrogativos o exclamativos; si las palabras cumplen los requisitos que se exigen para ser tildadas en este dispositivo (agudas terminadas en vocal, etc.) ellos proceden a trazar esta marca, con lo cual se logrará un primer resultado. Después, los expertos introducen mentalmente las palabras en un segundo dispositivo, el de la tilde de hiato acentual, que solo puede reconocer polisílabos con este tipo de hiato, y en cuanto este es identificado, ellos marcan la tilde sobre la vocal cerrada; así se obtiene un segundo resultado. Finalmente, las palabras son ingresadas a un tercer dispositivo, el de la tilde diacrítica, que solo puede reconocer los ocho monosílabos ya conocidos y todos los pronombres interrogativos y exclamativos; si los expertos encuentran cualquiera de estas palabras, no dudan en tildarlas; con ello se consigue un tercer resultado. En la Figura 2, se ha representado todo lo que hemos expuesto en estas últimas líneas.

Todas estas operaciones se producen a tal velocidad en las mentes de los expertos que incluso muchos de ellos podrían dudar de que efectivamente sea así como acontece el procesamiento de las palabras para ser tildadas. Es probable que alguien 
sostenga que se trata de un solo dispositivo en el que están todos los requisitos; pero, incluso de ser cierto esto último, de todos modos habría tres conjuntos de reglas bien diferenciados operando para grupos específicos de palabras, o sea, de todas maneras se tendría que mencionar que en ese único dispositivo habría tres filtros diferentes.

Debe tenerse presente que cada uno de los dispositivos supone el dominio de toda la información metalingüística que le corresponde (por ejemplo, en el último dispositivo, se sobrentiende que se debe distinguir entre un <qué> que es un pronombre interrogativo y un $<$ que $>$ que puede pertenecer a cualquier otra categoría palábrica excepto a la de los pronombres interrogativos o a la de los pronombres exclamativos).

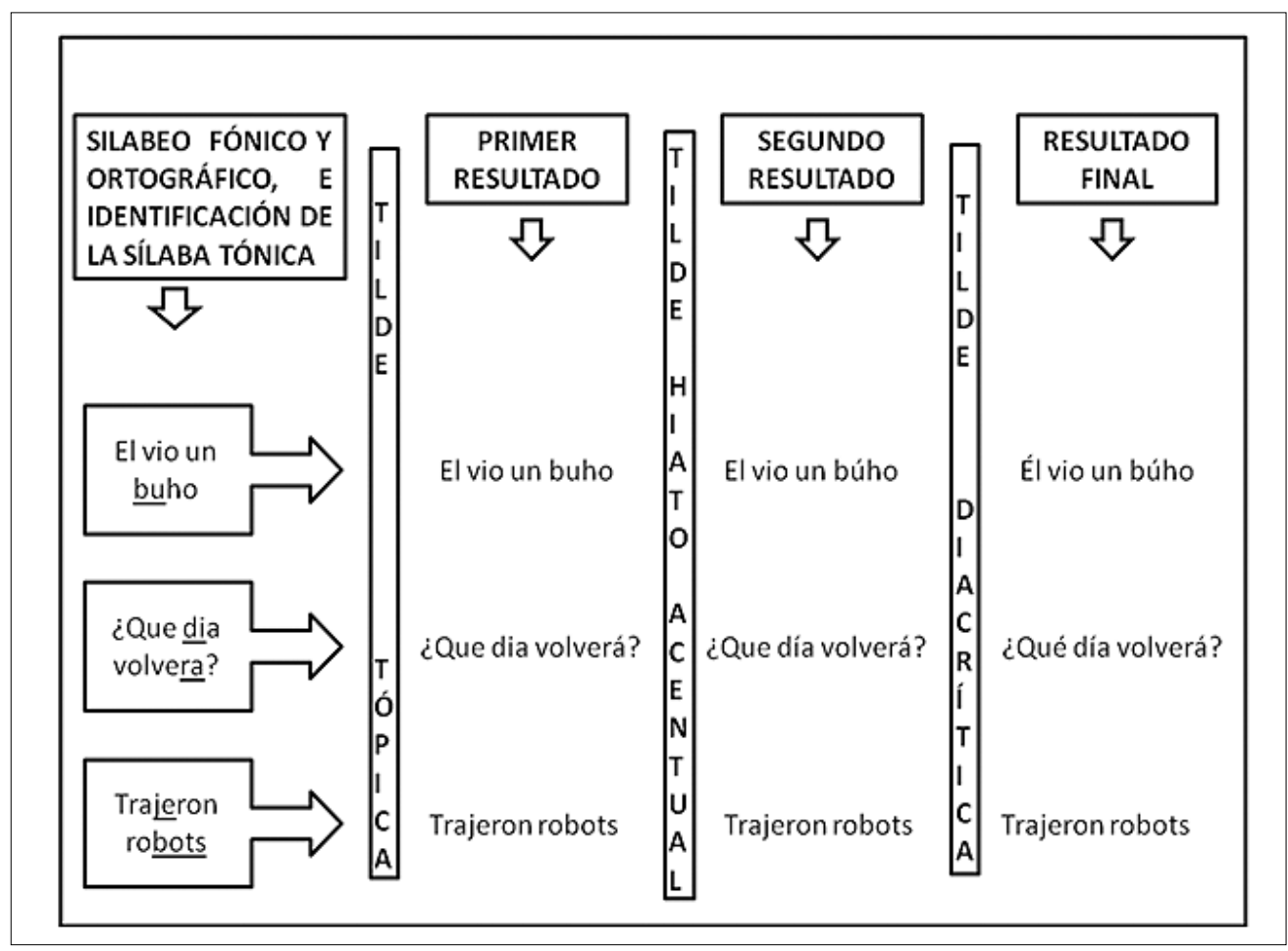

Figura 2. Procesamiento de palabras según la clase de tilde a que corresponden.

\section{CONCLUSIONES}

El ACT nos ha permitido mostrar que para poder usar correctamente la tilde, además de conocer las normas vigentes del acento ortográfico, se requiere lo siguiente: a) identificar la sílaba tónica; b) disociar el acento de la tilde; c) segmentar las palabras en sílabas ortográficas; d) distinguir entre polisílabos y monosílabos; e) diferenciar entre polisílabos con hiato acentual y polisílabos sin dicha secuencia; f) conocer las clases de palabras según el acento; g) reconocer palabras compuestas, extranjerismos, 
diptongos, triptongos y hiatos simples; h) identificar las siguientes clases de palabras: pronombres interrogativos, pronombres exclamativos, pronombres personales, nombres comunes, verbos, adverbios, determinantes; i) poseer competencia grafemática. Si esta información es determinante para el empleo correcto de la tilde, su tratamiento en la formación de los estudiantes resulta obligatorio; y la forma y la profundidad con que se aborden estos temas deben ser tales que realmente permitan su uso efectivo al momento de tildar. Al afirmar esto, estamos pensando en aquellos alumnos que no se equivocan al tildar los pronombres interrogativos en las oraciones interrogativas directas, pero sí lo hacen en las interrogativas indirectas (Pujol, 2005). Esto se puede observar en el ejemplo que este mismo autor reproduce: "El punto dos nos habla de como disminuye el precio de los libros, y como aumenta el precio de la radio" (Pujol, 2005: 33).

Si la programación curricular de la escuela no considera estos contenidos, estará condenada a obtener resultados negativos. Creemos que eso es lo que ocurre actualmente en varios países hispanohablantes.

Con el ACT, se ha mostrado también que para tildar sin error, se necesita ejecutar operaciones de regulación metacognitiva. La importancia de estas operaciones determina que el modelo de procesamiento de palabras presentado en la Figura 2 sea enseñado a los aprendices, puesto que es el que ponen en marcha quienes no se equivocan al aplicar la tilde.

Para propiciar el manejo de estas operaciones regulativas, se debe iniciar el tema del acento escrito señalando que hay tres clases de tilde y que cada una de ellas tiene reglas exclusivas para tipos específicos de palabras. Con ello se haría evidente la relación de complementariedad existente entre los tipos de tilde y las palabras que les corresponden. Al referirnos a las clases de tilde se deberá dejar de lado el rótulo 'tilde general', puesto que tal calificativo no le corresponde al constituir solo una clase con pertinencia para un grupo determinado de palabras. Y al detallar lo concerniente a cada clase de tilde se empezará con la que corresponde al hiato acentual, pues, como hemos mostrado aquí, es la que menor cantidad de conocimientos metalingüísticos exige. Pero es evidente que este último punto debe ser objeto de debate y en él deben considerarse otras variables.

De los hallazgos del presente trabajo se desprenden dos tareas: a) mostrar su adecuación empírica, lo cual que hemos empezado circunscribiéndonos a la tilde de hiato acentual; los resultados confirman la validez de nuestra contribución; b) incardinar el modelo presentado en la Figura 2 en alguno de los existentes sobre el procesamiento del código escrito en general, porque la tilde es solo una parte del código alfabético.

La forma como se ha logrado relacionar el nivel de competencia en el manejo de la tilde por un lado y el dominio y gestión de información metalingüística específica por otro, puede servir de pauta para estudiar otros fenómenos ortográficos. 


\section{REFERENCIAS BIBLIOGRÁFICAS}

Academia Boliviana de la Lengua. (2011). Presentación de la Ortografía. Boletín No 90 [en línea]. Disponible en: http://www.asale.org/Imagenes/ASALE_PDF/ BBolivia90.pdf

Ávila, F. (2002). Dónde va la tilde. Bogotá: Norma.

Barberá, V., Collado, J., Morató, J., Pellicer, C. \& Rizo, M. (2002). Didáctica de la ortografía. Barcelona: Ceac.

Banegas, D. \& Martínez, A. (s. d.). Sin Hache y con acento... sobre la ortografía en Español e Inglés [en línea]. Disponible en: http://www.isfd809esquel.com.ar/images/ archivos/sinhacheyconacento.pdf

Borzi, C. \& García, M. (2006). Errores ortográficos como recursos comunicativos. Didáctica. Lengua y Literatura [en línea]. Disponible en: http://revistas.ucm.es/index. $\mathrm{php/DIDA/article/view/DIDA0606110093A/19160}$

Cano, J. (1975). Lengua española. Arequipa: s. e.

Chipman, S., Schraagen, J. \& Shalin, V. (2000). Introduction to cognitive task analysis. En J. Schraagen, S. Chipman \& V. Shute (Eds.), Cognitive Task Analysis (pp. 3-23). Mahwah, NJ: Lawrence Erlbaum.

Crandall, B., Klein, G. \& Hoffman, R. (2006). Working minds. A practitioner's guide to cognitive task analysis. Londres: MIT.

Díaz, J. (2006). Habilidades metalingüisticas en niños alfabetizados de bajo nivel socioeconómico. Umbral Científico [en línea]. Disponible en: http://redalyc.uaemex.mx/ pdf/304/30400803.pdf

Duque, E. (s. f.). Acentuación gráfica en alumnos de la segunda etapa de educación básica [en línea]. Diponible en: http://servidor-opsu.tach.ula.ve/profeso/garcia_mar/ p_e_1_2/086_a_g.pdf

Fernández-Duque, D., Baird, J. \& Posner, M. (2000). Ejecutive attention and metacognitive regulation. Conciousness and Cognition, 9(2), 288-307.

Guitart, J. (2004). Sonido y sentido. Washington, D.C.: Georgetown University Press.

Lacon, N. \& Ortega, S. (2008). Cognición, metacognición y escritura. Revista Signos. Estudios de Lingüistica, 41(67), 231-255.

Martínez de Sousa, J. (2003). Algunos enfoques en la enseñanza de la ortografía [en línea]. Disponible en: http://www.martinezdesousa.net/ense_ortog.pdf 
Pérez, F. (1994). Ortografía actualizada. Santiago de Chile: Andrés Bello.

Portilla, L. (2003). El problema de la acentuación ortográfica de los estudiantes sanmarquinos [en línea]. Disponible en: http://www.cybertesis.edu.pe/sisbib/2003/portilla_ dl/pdf/portilla_dl.pdf

Pujol, M. (1999). Análisis de errores grafemáticos en textos libres de estudiantes de enseñanzas medias [en línea]. Disponible en: http://www.tdx.cat/bitstream/handle/10803/1283/ TESIS_COMPLETA.pdf?sequence $=1$

Pujol, M. (2005). La ortografía. En S. Torner \& P. Battaner (Eds.), El corpus PAAU 1992: Estudios descriptivos, textos y vocabulario (pp. 29-65). Barcelona: Institut Universitari de Lingüística Aplicada.

RAE. (2010). Ortografía de la lengua española. Madrid: Espasa.

RAE y Asociación de Academias de la Lengua Española. (2005). Diccionariopanhispánico de dudas. Bogotá: Santillana.

Rico, D. \& Vernon, S. (2011). El desarrollo de la localización del acento prosódico y la atildación en los niños. En K. Hess, G. Calderón, S. Vernon \& M. Alvarado (Coord.), Desarrollo lingüistico y cultura escrita (pp. 71-87). México D.F.: Universidad Autónoma de Querétaro.

Serna, J. (2004). Cómo escribir correctamente. Barcelona: Robinbook.

Sosa, F. (2007). Aplicación del acento ortográfico. Punto Cero, 12(14), 71-82.

Vernon, S. \& Pruneda, M. (2011). Secuencia didáctica para el aprendizaje de la acentuación gráfica española. En K. Hess, G. Calderón, S. Vernon \& M. Alvarado (Coord.), Desarrollo lingüístico y cultura escrita (pp. 89-106). México D.F.: Universidad Autónoma de Querétaro.

Villafana, J., Chacón, J., Conde, M. \& Quintanilla, R. (2009). Lenguaje. Lima: UNMSM-CEPRE.

Zayas, F. (1995). Ortografía y aprendizaje de la lengua escrita. Textos de Didáctica de la Lengua y de la Literatura [en línea]. Disponible en: http://www.xtec.cat/ ilopez15/ materials/expressioescrita/ortografiayaprendizajedelalenguaescrita.pdf 